\title{
Multiple non-negative solutions to a semilinear equation on Heisenberg group with indefinite nonlinearity
}

\author{
Lirong Huang ${ }^{1 *}$, Jianqing Chen ${ }^{2}$ and Eugénio M Rocha ${ }^{3}$
}

"Correspondence:
Irhuang515@126.com
${ }^{1}$ College of Mathematics and
Physics, Fujian Jiangxia University,
Fuzhou, 350108, P.R. China
Full list of author information is
available at the end of the article

available at the end of the article

\begin{abstract}
This paper is concerned with the existence and multiplicity of non-negative solutions to the semilinear equation $-\Delta_{H} u=K(\xi)|u|^{2^{\sharp}-2} u+\mu|\xi|_{H}^{\alpha} u$ in a bounded domain $\Omega \subset \mathbb{H}^{N}$ with Dirichlet boundary conditions. Here $\mathbb{H}^{N}$ is the Heisenberg group and $2 \sharp=2 q /(q-2)$ is the critical exponent of the Sobolev embedding on the Heisenberg group. The function $K(\xi)$ may be sign changing on $\Omega$. Using the variational method, we prove that this problem has at least two non-negative solutions provided $\mu, \alpha$, and $K(\xi)$ satisfy some conditions.
\end{abstract}

MSC: $35 J 20$

Keywords: multiple non-negative solutions; Heisenberg group; indefinite nonlinearity

\section{Introduction}

This paper is concerned with the existence and multiplicity of non-negative solutions to the semilinear equation on the Heisenberg group $\mathbb{H}^{N}$ of the form

$$
\begin{cases}-\Delta_{H} u=K(\xi)|u|^{2^{\sharp}}-2 u+\mu|\xi|_{H}^{\alpha} u & \text { in } \Omega, \\ u(\xi)=0 & \text { on } \partial \Omega,\end{cases}
$$

where $0 \in \Omega$ and $\Omega$ is a bounded domain with smooth boundary of the Heisenberg group $\mathbb{H}^{N}$. The $\Delta_{H}$ (see the definition below) is the Kohn Laplacian on the Heisenberg group. $2^{\sharp}$ is the critical exponent for the semilinear Dirichlet problem of the Kohn Laplacian, and the exponent $2 N /(N-2)$ is critical for the semilinear equation $-\Delta u=|u|^{\frac{2 N}{N-2}-2} u+h(u)$ in a domain of $\mathbb{R}^{N}$ with the Dirichlet boundary condition. The function $K(\xi) \in L^{\infty}(\Omega)$ and $K(\xi)=K_{+}-K_{-}$with $K_{+}=\max \{K(\xi), 0\} \neq 0$ and $K_{-}=\max \{-K(\xi), 0\} \neq 0$, which is why we use the terms indefinite nonlinearity in the title.

We start with some basic notions (see e.g. [1]). The Heisenberg group $\mathbb{H}^{N}$ is identified with $\mathbb{R}^{2 N+1}$ under the following group composition: for all $\xi=(x, y, t)$ and $\xi^{\prime}=\left(x^{\prime}, y^{\prime}, t^{\prime}\right)$,

$$
\xi \circ \xi^{\prime}=\left(x+x^{\prime}, y+y^{\prime}, t+t^{\prime}+2\left(x \cdot y^{\prime}-x^{\prime} \cdot y\right)\right)
$$

(c) 2015 Huang et al. This article is distributed under the terms of the Creative Commons Attribution 4.0 International License (http://creativecommons.org/licenses/by/4.0/), which permits unrestricted use, distribution, and reproduction in any medium, provided you give appropriate credit to the original author(s) and the source, provide a link to the Creative Commons license, and indicate if changes were made. 
where ' '. 'denotes the inner product in $\mathbb{R}^{N}$. For any $\xi \in \mathbb{H}^{N}$, the left translations on $\mathbb{H}^{N}$ is defined by

$$
\tau_{\xi}: \mathbb{H}^{N} \rightarrow \mathbb{H}^{N}, \quad \tau_{\xi}\left(\xi^{\prime}\right)=\xi \circ \xi^{\prime} .
$$

For $\lambda>0$, a family of dilation on $\mathbb{H}^{N}$ is defined by

$$
\delta_{\lambda}: \mathbb{H}^{N} \rightarrow \mathbb{H}^{N}, \quad \delta_{\lambda}(x, y, t)=\left(\lambda x, \lambda y, \lambda^{2} t\right)
$$

The homogeneous dimension of $\mathbb{H}^{N}$ is $q=2 N+2$. For $\xi \in \mathbb{H}^{N},|\xi|_{H}$ is the intrinsic distance of the point $\xi$ to the origin, namely

$$
|\xi|_{H}=\left(\sum_{j=1}^{N}\left(x_{j}^{2}+y_{j}^{2}\right)^{2}+t^{2}\right)^{\frac{1}{4}}
$$

The Kohn Laplacian $\Delta_{H}$ on $\mathbb{H}^{N}$ is defined as

$$
\Delta_{H}=\sum_{j=1}^{N}\left(X_{j}^{2}+Y_{j}^{2}\right),
$$

where

$$
X_{j}=\frac{\partial}{\partial x_{j}}+2 y_{j} \frac{\partial}{\partial t}, \quad Y_{j}=\frac{\partial}{\partial y_{j}}-2 x_{j} \frac{\partial}{\partial t} .
$$

For every $u \in C_{0}^{\infty}(\Omega)$, the subelliptic gradient is defined as

$$
\nabla_{H} u=\left(X_{1} u, \ldots, X_{N} u, Y_{1} u, \ldots, Y_{N} u\right) .
$$

The closure of $C_{0}^{\infty}(\Omega)$ under the norm $\int_{\Omega}\left|\nabla_{H} \cdot\right|^{2} d \xi$ is denoted by $S_{0}^{1,2}(\Omega)$. From [2, 3], we also know that the following Sobolev type inequality holds: there exists $C_{q}>0$ such that

$$
|u|_{2 q /(q-2)} \leq C_{q}\|u\|_{S_{0}^{1,2}\left(\mathbb{H}^{N}\right)} \quad \text { for all } u \in S_{0}^{1,2}\left(\mathbb{H}^{N}\right)
$$

where $|\cdot|_{2 q /(q-2)}$ is the norm in $L^{2 q /(q-2)}$. The number $2 q /(q-2):=2^{\sharp}$ is the critical Sobolev exponent, since for a bounded domain $\Omega$ and $2<p<2 q /(q-2)$, the $S_{0}^{1,2}(\Omega)$ is compactly embedded into $L^{p}(\Omega)$, while this inclusion is only continuous if $p=2 q /(q-2)$.

There are several papers studying the existence and nonexistence of solutions of semilinear equations with Kohn Laplacian in the past two decades. For instance, Citti [4] studies the equation

$$
-\triangle_{H} u+a u=u^{\frac{q+2}{q-2}} \quad \text { in } \Omega, \quad u=0 \quad \text { on } \partial \Omega,
$$

where $\Omega$ is a smooth bounded domain in $\mathbb{H}^{N}$. Since (1.3) involves a nonlinearity of critical growth, Citti [4] has proven a representation formula for the Palais-Smale sequence and then proved the existence of one non-negative solution of (1.3) under suitable conditions 
of $a$. Some results of Liouville type for semilinear equations on the Heisenberg group have been studied by Birindelli et al. [5, 6]. Uguzzoni [7] has proven a nonexistence theorem for a semilinear Dirichlet problem involving critical nonlinearity on the half space of the Heisenberg group. Yamabe-type equations on the Heisenberg group have been studied in [8-10]. Garofalo et al. [11] have studied some other existence and nonexistence of solutions for the Kohn Laplace semilinear equations. Other existence and nonexistence results for elliptic problems on Heisenberg have been studied in [12-20]. Very recently, Han et al. [21] have proven a class of Hardy-Sobolev type inequalities on H-type group and got the existence of a nontrivial solution for a related equation. A multiplicity result related to noncontractible domain has been studied in [22]. But we do not see any multiplicity results as regards the semilinear equation with critical exponent on the Heisenberg group with general bounded domain.

The purpose of the present paper is to prove that under suitable assumptions on $K(\xi)$ and $\mu$, the problem under consideration has at least two non-negative solutions. Here and subsequently, we say that $u \in S_{0}^{1,2}(\Omega)$ is a solution of (1.1) if and only if for any $\psi \in C_{0}^{\infty}(\Omega)$, we have

$$
\int\left(\nabla_{H} u \nabla_{H} \psi-\mu|\xi|_{H}^{\alpha} u \psi\right) d \xi-\int K(\xi)|u|^{2^{\sharp}-2} u \psi d \xi=0 .
$$

$u \in S_{0}^{1,2}(\Omega)$ is said to be a non-negative solution of (1.1) if $u$ is a solution and $u \geq 0$ but $u \neq 0$. According to the Sobolev inequality [23], we know that the functional

$$
L(u)=\frac{1}{2} \int\left(\left|\nabla_{H} u\right|^{2}-\mu|\xi|_{H}^{\alpha}|u|^{2}\right) d \xi-\frac{1}{2^{\sharp}} \int K(\xi)|u|^{2^{\sharp}} d \xi
$$

is well defined and $C^{1}$ on $S_{0}^{1,2}(\Omega)$. Note that from Lemma 2.4 (see Section 2) the eigenvalue problem

$$
-\Delta_{H} u=\mu|\xi|_{H}^{\alpha} u, \quad u \in S_{0}^{1,2}(\Omega)
$$

has a sequence of eigenvalues $0<\mu_{1}<\mu_{2} \leq \mu_{3} \leq \cdots \leq \mu_{m}<\cdots, \mu_{m} \rightarrow \infty$ as $m \rightarrow \infty$, with the first eigenvalue $\mu_{1}$ simple, and all the eigenvalues are of finite multiplicity. Up to a normalization, the first eigenfunction $e_{1}$ corresponding to $\mu_{1}$ is non-negative. The basic assumptions are:

(A1) $0<K(0)=\max _{\xi \in \bar{\Omega}}|K(\xi)|$ and there is $R>0$ such that for $\xi \in B(0,2 R)$,

$$
K(\xi)=K(0)+O\left(|\xi|_{H}^{\beta}\right) \text { with } 2+\alpha<\beta<q
$$

(A2) $\int_{\Omega} K(\xi) e_{1}^{2^{*}} d \xi<0$, where $e_{1}>0$ is as mentioned before.

Our main results are

Theorem 1.1 Suppose that (A1) holds. If $\mu \in\left(0, \mu_{1}\right)$, then (1.1) has at least one nonnegative solution.

Theorem 1.2 Suppose that (A1) and (A2) hold.

(1) If $\mu=\mu_{1}$, then (1.1) has at least one non-negative solution;

(2) if $0<\alpha<\frac{q}{2}-3$, then there is $\mu_{*}>\mu_{1}$, such that for any $\mu \in\left(\mu_{1}, \mu_{*}\right)$, (1.1) has at least two non-negative solutions. 
The proofs of Theorem 1.1 and Theorem 1.2 are based on critical point theory. Our idea originates from $[24,25]$. More precisely, we will minimize the functional $L$ over a suitable subset of $S_{0}^{1,2}(\Omega)$ according to the range of $\mu$. However, since the embedding $S_{0}^{1,2}(\Omega) \hookrightarrow L^{2^{\sharp}}(\Omega)$ is not compact, the standard minimization argument cannot be applied directly. We have to estimate the minimum level of the functional $L$ carefully such that it is contained in the range where the Palais-Smale ((PS) for short, see Definition 2.3) condition holds. On getting one non-negative solution, we can modify the argument from [26]. However, in order to get the existence of a second solution, one needs a priori estimate about the property of the first solution. In [25], Drabek et al. overcome this difficulty by the fact that any solutions belong to $L^{\infty}$. While in [24], the author has managed to get two positive solutions by establishing an exact local behavior of positive solutions near singularity. But for the semilinear equation on Heisenberg group, the operator $-\Delta_{H}$ is degenerate. It is not easy to get the boundedness of the solution to semilinear equation with critical exponent. One of our contributions here is to estimate the integrals in a suitable way and do the energy estimates without the boundedness of the solution.

This paper is organized as follows. Section 2 contains some preliminaries. Particular attention is focused on several integral estimates for solutions of (1.1), which will play an important role in the study of multiple solutions of (1.1). The third and fourth sections are devoted to the proofs of Theorem 1.1 and Theorem 1.2, respectively.

\section{Preliminaries}

Throughout this paper, $C, C_{j}(j=1,2, \ldots)$ will denote various positive constants whose exact value are not important. The dual space of a Banach space $E$ is denoted by $E^{*}$. By $|\cdot|_{p}$ we denote the norm in $L^{p}(\Omega) . S_{0}^{1,2}\left(\mathbb{H}^{N}\right)$ is the closure of $C_{0}^{\infty}\left(\mathbb{H}^{N}\right)$ under the norm of $\int_{\mathbb{H}^{N}}\left|\nabla_{H} \cdot\right|^{2} d x . B(\xi, r)$ is a ball centered at $\xi$ with radius $r . O\left(\varepsilon^{m}\right)$ denotes $\left|O\left(\varepsilon^{m}\right)\right| / \varepsilon^{m} \leq C$ and $o\left(\varepsilon^{m}\right)$ denotes $\left|o\left(\varepsilon^{m}\right)\right| / \varepsilon^{m} \rightarrow 0$ as $\varepsilon \rightarrow 0$. All integrals are taken over $\Omega$ unless stated otherwise. The following minimization problem will be useful in what follows:

$$
S=\inf \left\{\int_{\mathbb{H}^{N}}\left|\nabla_{H} u\right|^{2} d \xi ; u \in S_{0}^{1,2}\left(\mathbb{H}^{N}\right), \int_{\mathbb{H}^{N}}|u|^{2^{\sharp}} d \xi=1\right\} .
$$

Jerison et al. [23] have proven that $S$ is achieved by

$$
\omega(x, y, t)=\frac{C_{0}}{\left(t^{2}+\left(1+|x|^{2}+|y|^{2}\right)^{2}\right)^{\frac{q-2}{4}}}
$$

with suitable positive constant $C_{0}$. Moreover, $\omega(x, y, t)$ satisfies

$$
-\Delta_{H} u(\xi)=|u(\xi)|^{2^{\sharp}-2} u(\xi), \quad \xi \in \mathbb{H}^{N}, u \in S_{0}^{1,2}\left(\mathbb{H}^{N}\right) .
$$

All non-negative solutions of (2.1) are of the form

$$
\omega_{\lambda, \xi^{\prime}}=\lambda^{\frac{q-2}{2}} \omega\left(\delta_{\lambda}\left(\tau_{\xi^{\prime}}^{-1}\right)\right), \quad \lambda>0, \xi^{\prime} \in \mathbb{H}^{N} .
$$

Moreover,

$$
\int_{\mathbb{H}^{N}}\left|\nabla_{H} \omega_{\lambda, \xi^{\prime}}\right|^{2} d \xi=\int_{\mathbb{H}^{N}}\left|\omega_{\lambda, \xi^{\prime}}\right|^{2^{\sharp}} d \xi=S^{\frac{q}{2}} .
$$


Define a cut-off function $\phi(\xi)$ and denote $w_{\lambda}(\xi)=\lambda^{\frac{q-2}{2}} \omega\left(\delta_{\lambda}(\xi)\right)$. Setting $v_{\lambda}(\xi):=\phi(\xi) w_{\lambda}(\xi)$, one can have from direct computations (see e.g. [4]) that as $\lambda \rightarrow+\infty$,

$$
\int\left|\nu_{\lambda}\right|^{\sharp^{\sharp}} d \xi=S^{\frac{q}{2}}+O\left(\lambda^{-q}\right)
$$

and

$$
\int\left|\nabla_{H} v_{\lambda}\right|^{2} d \xi=S^{\frac{q}{2}}+O\left(\lambda^{-(q-2)}\right)
$$

Using this idea, we can deduce the following lemma, which will play an important role in the proofs of Theorem 1.1 and Theorem 1.2.

Lemma 2.1 Let $v_{\lambda}$ be defined as above. If $2+\alpha<\beta<q$, then as $\lambda \rightarrow+\infty$,

$$
\begin{aligned}
& \int|\xi|_{H}^{\beta}\left|\nu_{\lambda}\right|^{2^{\sharp}} d \xi=O\left(\lambda^{-\beta}\right) ; \\
& \int|\xi|_{H}^{\alpha}\left|v_{\lambda}\right|^{2} d \xi=O\left(\lambda^{-(\alpha+2)}\right) .
\end{aligned}
$$

Proof Keep the definition of $v_{\lambda}$ in mind. We have

$$
\begin{aligned}
\int_{\Omega}|\xi|_{H}^{\beta}\left|\nu_{\lambda}\right|^{2^{\sharp}} d \xi & =\int_{|\xi|_{H}<2 R}|\xi|_{H}^{\beta}\left(\lambda^{\frac{q-2}{2}} \omega\left(\delta_{\lambda}(\xi)\right)\right)^{2^{\sharp}} d \xi \\
& =\lambda^{-\beta} \int_{|\eta|_{H}<2 \lambda R}|\eta|_{H}^{\beta}(\omega(\eta))^{2^{\sharp}} d \eta \\
& =\lambda^{-\beta}\left(\int_{|\eta|_{H}<1}|\eta|_{H}^{\beta}(\omega(\eta))^{2^{\sharp}} d \eta+\int_{1<|\eta|_{H}<2 \lambda R}|\eta|_{H}^{\beta}(\omega(\eta))^{2^{\sharp}} d \eta\right) \\
& \leq \lambda^{-\beta}\left(C+\int_{1}^{2 \lambda R} \rho^{\beta-q-1} d \rho\right) \\
& =O\left(\left(\lambda^{-1}\right)^{\beta}\right)+O\left(\left(\lambda^{-1}\right)^{q}\right)=O\left(\left(\lambda^{-1}\right)^{\beta}\right) \quad \text { for } \lambda \text { large enough, }
\end{aligned}
$$

where we have used the assumption of $\beta<q$. Similarly, we have

$$
\begin{aligned}
\int_{\Omega}|\xi|_{H}^{\alpha}\left|\nu_{\lambda}\right|^{2} d \xi & =\int_{|\xi|_{H}<2 R}|\xi|_{H}^{\alpha} w_{\lambda}^{2}\left(\delta_{\lambda}(\xi)\right) d \xi \\
& =\lambda^{-2-\alpha} \int_{|\eta|_{H}<2 \lambda R}|\eta|_{H}^{\alpha} \omega^{2}(\eta) d \eta \\
& =\lambda^{-2-\alpha}\left(\int_{|\eta|_{H}<1}|\eta|_{H}^{\alpha} \omega^{2}(\eta) d \eta+\int_{1<|\eta|_{H}<2 \lambda R}|\eta|_{H}^{\alpha} \omega^{2}(\eta) d \eta\right) \\
& \leq \lambda^{-2-\alpha}\left(C+\int_{1}^{2 \lambda R} \rho^{4-q+\alpha-1} d \rho\right) \\
& =O\left(\left(\lambda^{-1}\right)^{2+\alpha}\right)+O\left(\left(\lambda^{-1}\right)^{q-2}\right) \quad \text { for } \lambda \text { large enough. }
\end{aligned}
$$

Therefore $0<\alpha<q-4$ implies that for $\lambda$ large enough,

$$
\int_{\Omega}|\xi|_{H}^{\alpha}\left|v_{\lambda}\right|^{2} d \xi=O\left(\lambda^{-(2+\alpha)}\right)
$$


The proof is complete.

Next, we prove a regularity result for the solutions of (1.1). The idea originates from Brezis-Kato [27]; see also Struwe [28]. The following lemma will play a key role in the process of studying a second non-negative solution of (1.1).

Lemma 2.2 If $u \in S_{0}^{1,2}(\Omega)$ is a solution of $(1.1)$, then $u \in L^{r}(\Omega)$ for each $r \in(1,+\infty)$.

Proof Since $u$ is a weak solution of (1.1), we test the equation with a test function $\varphi=$ $u \min \left\{|u|^{2 s}, m^{2}\right\}$, where $s \geq 0$ and $m>1$. Integrating by parts we obtain

$$
\begin{aligned}
\int \nabla_{H} u \nabla_{H}\left(u \min \left\{|u|^{2 s}, m^{2}\right\}\right) d \xi= & \int|u|^{2^{\sharp}} \min \left\{|u|^{2 s}, m^{2}\right\} d \xi \\
& +\mu \int|\xi|_{H}^{\alpha} u^{2} \min \left\{|u|^{2 s}, m^{2}\right\} d \xi .
\end{aligned}
$$

For each sufficiently large $M>0$, we deduce that

$$
\begin{aligned}
& \int\left|\nabla_{H}\left(u \min \left\{|u|^{s}, m\right\}\right)\right| d \xi \\
& \leq(2 s+2) \int|u|^{2^{\sharp}} \min \left\{|u|^{2 s}, m^{2}\right\} d \xi+C \int u^{2} \min \left\{|u|^{2 s}, m^{2}\right\} d \xi \\
& \quad=(2 s+2) \int_{|u| \leq M}|u|^{2^{\sharp}} \min \left\{|u|^{2 s}, m^{2}\right\} d \xi+C \int u^{2} \min \left\{|u|^{2 s}, m^{2}\right\} d \xi, \\
& (2 s+2) \int_{|u|>M}|u|^{2^{\sharp}} \min \left\{|u|^{2 s}, m^{2}\right\} d \xi \\
& \leq(2 s+2) \operatorname{meas}(\Omega) M^{2^{\sharp}+2 s}+C \int u^{2} \min \left\{|u|^{2 s}, m^{2}\right\} d \xi, \\
& (2 s+2)\left(\int_{|u|>M}|u|^{2^{\sharp}} d \xi\right)^{\frac{2^{\sharp}-2}{2^{\sharp}}}\left(\int\left(u \min \left\{|u|^{2 s}, m^{2}\right\} d\right)^{2^{\sharp}} d \xi\right)^{\frac{2}{2^{\sharp}}} \\
& \leq C+\frac{1}{2} \int\left|\nabla_{H}\left(u \min \left\{|u|^{s}, m\right\}\right)\right|^{2} d \xi+C \int u^{2} \min \left\{|u|^{2 s}, m^{2}\right\} d \xi,
\end{aligned}
$$

which implies that

$$
\int\left|\nabla_{H}\left(u \min \left\{|u|^{s}, m\right\}\right)\right| d \xi \leq 4(s+1) \operatorname{meas}(\Omega) M^{2^{\sharp}+2 s}+C_{1} \int u^{2} \min \left\{|u|^{2 s}, m^{2}\right\} d \xi .
$$

Letting $m \rightarrow+\infty$, we obtain

$$
\int\left|\nabla_{H}\left(u|u|^{s}\right)\right|^{2} d \xi \leq 4(s+1) \operatorname{meas}(\Omega) M^{2^{\sharp}+2 s}+C_{1} \int|u|^{2(s+1)} d \xi .
$$

Now iterate, letting $s_{0}=0, s_{j}+1=\left(s_{j-1}+1\right) \frac{q}{q-2}$, if $j \geq 1$, to obtain the conclusion.

We end these preliminaries by the definition of the $(P S)$ conditions and an additional lemma. 
Definition 2.3 Let $c \in \mathbb{R}, E$ be a Banach space and $I \in C^{1}(E, \mathbb{R})$. We say that $I$ satisfies $(P S)_{c}$ condition, if any sequence $\left(u_{n}\right)_{n \in \mathbb{N}}$ in $E$ such that $I\left(u_{n}\right) \rightarrow c$ and $I^{\prime}\left(u_{n}\right) \rightarrow 0$ has a convergent subsequence. If this holds for every $c \in \mathbb{R}$, we say that $I$ satisfies the $(P S)$ condition.

Lemma 2.4 Let $\Omega \subset \mathbb{H}^{N}$ be a bounded open domain with smooth boundary. Then $S_{0}^{1,2}(\Omega)$ is continuously and compactly embedded to $L^{2}\left(\Omega,|\xi|_{H}^{\alpha} d \xi\right)$.

Proof Since $\alpha>0$, we can get the conclusion by a combination of [29], Lemma 3.2, and [30], Lemma 2.6.

\section{Existence of a non-negative solution}

In this section, we will prove Theorem 1.1. The $0<\mu<\mu_{1}$ and (A1) will be assumed throughout this section. Define another functional

$$
G(u)=\int\left|\nabla_{H} u\right|^{2} d \xi-\mu \int|\xi|_{H}^{\alpha}|u|^{2} d \xi-\int K(\xi)|u|^{2^{\sharp}} d \xi, \quad u \in S_{0}^{1,2}(\Omega),
$$

and denote the Nehari set

$$
\mathcal{N}_{\mu}=\left\{u \in S_{0}^{1,2}(\Omega) \backslash\{0\}: G(u)=0\right\} .
$$

We have first of all the following.

Lemma 3.1 There is $\rho_{0}>0$ such that $\|u\| \geq \rho_{0}$ for all $u \in \mathcal{N}_{\mu}$.

Proof For any $u \in \mathcal{N}_{\mu}$, the assumption (A1) and the Sobolev inequality imply that

$$
\|u\|^{2}-\mu \int|\xi|_{H}^{\alpha}|u|^{2} d \xi=\int K(\xi)|u|^{2^{\sharp}} d \xi \leq K(0)|u|_{2^{\sharp}}^{2^{\sharp}} \leq K(0) S^{-\frac{2^{\sharp}}{2}}\|u\|^{2^{\sharp}} .
$$

Therefore $\left(1-\frac{\mu}{\mu_{1}}\right)\|u\|^{2} \leq K(0) S^{-\frac{2^{\sharp}}{2}}\|u\|^{2^{\sharp}}$. Hence we can choose

$$
\rho_{0}=\left(\left(1-\frac{\mu}{\mu_{1}}\right) K(0)^{-1} S^{\frac{2 \sharp}{2}}\right)^{\frac{1}{2 \sharp-2}}
$$

such that Lemma 3.1 holds.

Note that for any $u \in \mathcal{N}_{\mu}$,

$$
L(u)=\frac{1}{q}\left(\|u\|^{2}-\mu \int|\xi|_{H}^{\alpha}|u|^{2} d \xi\right)=\frac{1}{q} \int K(\xi)|u|^{2^{\sharp}} d \xi .
$$

We define

$$
d_{1}=\inf _{u \in \mathcal{N}_{\mu}} L(u)
$$

From Lemma 3.1, one sees immediately that there is a positive constant $C_{0}$ such that $c_{1} \geq$ $C_{0}>0$. Next, we have the following lemma. 
Lemma 3.2 There is a sequence $\left(u_{n}\right)_{n \in \mathbb{N}} \subset \mathcal{N}_{\mu}$ such that

$$
L\left(u_{n}\right) \rightarrow c_{1}, \quad L^{\prime}\left(u_{n}\right) \rightarrow 0 \quad \text { in }\left(S_{0}^{1,2}(\Omega)\right)^{*} .
$$

Proof Let $\left(\tilde{u}_{n}\right)_{n \in \mathbb{N}} \subset \mathcal{N}_{\mu}$ be a minimizing sequence of (3.1). By the Ekeland variational principle, we can find a sequence $\left(u_{n}\right)_{n \in \mathbb{N}} \subset \mathcal{N}_{\mu}$ such that

$$
L\left(u_{n}\right) \rightarrow c_{1},\left.\quad L^{\prime}\right|_{\mathcal{N}_{\mu}}\left(u_{n}\right) \rightarrow 0,
$$

where $\left.L^{\prime}\right|_{\mathcal{N}_{\mu}}$ is the derivative of $L$ restricted to $\mathcal{N}_{\mu}$. The Lagrange multiplier rule implies that there is $a_{n} \in \mathbb{R}$ such that

$$
L^{\prime}\left(u_{n}\right)-a_{n} G^{\prime}\left(u_{n}\right) \rightarrow 0 \quad \text { and } \quad\left\langle L^{\prime}\left(u_{n}\right), u_{n}\right\rangle=a_{n}\left\langle G^{\prime}\left(u_{n}\right), u_{n}\right\rangle
$$

Since $u_{n} \in \mathcal{N}_{\mu}$, one deduces that $\left\langle G^{\prime}\left(u_{n}\right), u_{n}\right\rangle \neq 0$ and then $\left\langle L^{\prime}\left(u_{n}\right), u_{n}\right\rangle=0$. Hence $a_{n}=0$. The conclusion follows.

Lemma 3.3 Let $\left(u_{n}\right)_{n \in \mathbb{N}} \subset \mathcal{N}_{\mu}$ be as in Lemma 3.2. If $c_{1}<\frac{1}{q} \frac{S^{\frac{q}{2}}}{|K|_{\infty}^{(q-2) / 2}}$, then $\left(u_{n}\right)_{n \in \mathbb{N}}$ possesses a convergent subsequence in $S_{0}^{1,2}(\Omega)$.

Proof The proof can be proceeded by following the same lines as [24], Lemma 3.3; see also Drabek [25].

Lemma 3.4 Under the assumptions of Theorem 1.1, we have

$$
c_{1}<\frac{1}{q} \frac{S^{\frac{q}{2}}}{|K|_{\infty}^{\frac{q-2}{2}}} .
$$

Proof It suffices to find some $u \in \mathcal{N}_{\mu}$ such that $L(u)<\frac{1}{q} \frac{S^{\frac{q}{2}}}{|K|_{\infty}^{(q-2) / 2}}$. Let $v_{\lambda}$ be defined as in Section 2, we have from direct computation that there is a $t_{0}$ with

$$
t_{0}=\left(\frac{\left\|v_{\lambda}\right\|^{2}-\mu \int|\xi|_{H}^{\alpha}\left|\nu_{\lambda}\right|^{2} d \xi}{\int K(\xi)\left|v_{\lambda}\right|^{2^{\sharp}} d \xi}\right)^{\frac{q-2}{4}}
$$

such that $t_{0} v_{\lambda} \in \mathcal{N}_{\mu}$. Moreover, we obtain from (2.2), (2.3), and Lemma 2.1

$$
\begin{aligned}
L\left(t_{0} v_{\lambda}\right) & =\frac{1}{q} t_{0}^{2}\left(\left\|v_{\lambda}\right\|^{2}-\mu \int|\xi|_{H}^{\alpha}\left|\nu_{\lambda}\right|^{2} d \xi\right) \\
& =\frac{1}{q}\left(\left\|v_{\lambda}\right\|^{2}-\mu \int|\xi|_{H}^{\alpha}\left|\nu_{\lambda}\right|^{2} d \xi\right)^{\frac{q}{2}}\left(\int K(\xi)\left|v_{\lambda}\right|^{2^{\sharp}} d \xi\right)^{\frac{2-q}{2}} \\
& =\frac{1}{q}\left(S^{\frac{q}{2}}+O\left(\left(\lambda^{-1}\right)^{q-2}\right)-O\left(\left(\lambda^{-1}\right)^{\alpha+2}\right)\right)^{\frac{q}{2}}\left(|K|_{\infty} S^{\frac{q}{2}}+O\left(\left(\lambda^{-1}\right)^{\beta}\right)+O\left(\left(\lambda^{-1}\right)^{q}\right)\right)^{\frac{2-q}{2}} \\
& <\frac{1}{q} \frac{S^{\frac{q}{2}}}{|K|_{\infty}^{\frac{q-2}{2}}} \text { for } \lambda \text { large enough, }
\end{aligned}
$$

where we have used the fact that $2+\alpha<\beta$ and $2+\alpha<q-2$. 
Proof of Theorem 1.1 Combining Lemmas 3.1-3.4, we have an $w_{1} \in \mathcal{N}_{\mu}$ which leads to $c_{1}$. Since if $\left(u_{n}\right)_{n \in \mathbb{N}}$ minimize $L$ over $\mathcal{N}_{\mu}$, then so does $\left(\left|u_{n}\right|\right)_{n \in \mathbb{N}}$, we can assume that $w_{1}$ is a non-negative critical point of $L$. Hence $w_{1}$ is a non-negative solution of (1.1).

\section{Existence results for $\mu \geq \mu_{1}$}

In this section, we will prove Theorem 1.2. The multiplicity result can be obtained by minimizing $L$ over different subset of $S_{0}^{1,2}(\Omega)$. The idea originates from Drabek et al. [25], where the authors study an indefinite problem in the classical Euclidean space $\mathbb{R}^{N}$, and some refinement from Chen [24], where the author studied an indefinite problem with singular term. The additional assumption (A2) will hold throughout this section. Since we will prove Theorem 1.2 for different $\mu$, we denote $L_{\mu} \equiv L$ from now on. Define the following Nehari type set:

$$
\mathcal{M}_{\mu}=\left\{u \in S_{0}^{1,2}(\Omega): G(u) \equiv\left\langle L_{\mu}^{\prime}(u), u\right\rangle=0\right\} .
$$

We further split $\mathcal{M}_{\mu}$ into three disjoint subsets,

$$
\begin{aligned}
\mathcal{M}_{\mu}^{+} & =\left\{u \in \mathcal{M}_{\mu}:\left\langle G^{\prime}(u), u\right\rangle>0\right\} \\
& =\left\{u \in \mathcal{M}_{\mu}:\|u\|^{2}-\mu \int|\xi|_{H}^{\alpha}|u|^{2} d \xi>\left(2^{\sharp}-1\right) \int K(\xi)|u|^{2^{\sharp}} d \xi\right\} \\
& =\left\{u \in \mathcal{M}_{\mu}: \int K(\xi)|u|^{\sharp} d \xi<0\right\}, \\
\mathcal{M}_{\mu}^{0} & =\left\{u \in \mathcal{M}_{\mu}:\left\langle G^{\prime}(u), u\right|=0\right\} \\
& =\left\{u \in \mathcal{M}_{\mu}:\|u\|^{2}-\mu \int|\xi|_{H}^{\alpha}|u|^{2} d \xi=\left(2^{\sharp}-1\right) \int K(\xi)|u|^{2^{\sharp}} d \xi\right\} \\
& =\left\{u \in \mathcal{M}_{\mu}: \int K(\xi)|u|^{\sharp} d \xi=0\right\},
\end{aligned}
$$

and

$$
\begin{aligned}
\mathcal{M}_{\mu}^{-} & =\left\{u \in \mathcal{M}_{\mu}:\left\langle G^{\prime}(u), u\right\rangle<0\right\} \\
& =\left\{u \in \mathcal{M}_{\mu}:\|u\|^{2}-\mu \int|\xi|_{H}^{\alpha}|u|^{2} d \xi<\left(2^{\sharp}-1\right) \int K(\xi)|u|^{2^{\sharp}} d \xi\right\} \\
& =\left\{u \in \mathcal{M}_{\mu}: \int K(\xi)|u|^{2^{\sharp}} d \xi>0\right\} .
\end{aligned}
$$

Remark 4.1 Now some remarks are in order.

(1) $K_{+} \neq 0$ implies that $\mathcal{M}_{\mu}^{-} \neq \emptyset$. Indeed, since

$$
\left\|\nu_{\lambda}\right\|^{2}-\mu \int|\xi|_{H}^{\alpha}\left|\nu_{\lambda}\right|^{2} d \xi=S^{\frac{q}{2}}+O\left(\left(\lambda^{-1}\right)^{q-2}\right)-O\left(\left(\lambda^{-1}\right)^{2+\alpha}\right)>0
$$

for $\lambda$ large enough, we know that $t_{0} v_{\lambda} \in \mathcal{M}_{\mu}^{-}$with

$$
t_{0}=\left(\frac{\left\|v_{\lambda}\right\|^{2}-\mu \int|\xi|_{H}^{\alpha}\left|\nu_{\lambda}\right|^{2} d \xi}{\int K(\xi)\left|v_{\lambda}\right|^{2^{\sharp}} d \xi}\right)^{\frac{(q-2)}{4}} .
$$


(2) $\mathcal{M}_{\mu}$ and $\mathcal{M}_{\mu}^{0}$ are closed in $S_{0}^{1,2}(\Omega)$.

(3) For $\mu \in\left(0, \mu_{1}\right], \mathcal{M}_{\mu}^{+}=\emptyset$. However, for $\mu>\mu_{1}, \mathcal{M}_{\mu}^{+} \neq \emptyset$. Indeed, we obtain from $\int K(\xi) e_{1}^{2^{\sharp}} d \xi<0$ and direct computation

$$
\left(\frac{\left\|e_{1}\right\|^{2}-\mu \int|\xi|_{H}^{\alpha}\left|e_{1}\right|^{2} d \xi}{\int K(\xi) e_{1}^{2^{\sharp}} d \xi}\right)^{\frac{q-2}{4}} e_{1} \in \mathcal{M}_{\mu}^{+} .
$$

In view of Remark 4.1, we will prove Theorem 1.2 in the following outline. For $\mu=\mu_{1}$, we will minimize $L_{\mu}$ on $\mathcal{M}_{\mu_{1}}^{-}$and prove the minimizer can be achieved and can be chosen to non-negative. For $\mu>\mu_{1}$, we will minimize $L_{\mu}$ on $\mathcal{M}_{\mu}^{+}$and $\mathcal{M}_{\mu}^{-}$, respectively and show the minimizers exist. Then we will get two non-negative solutions of (1.1). The following lemmas are useful in what follows.

Lemma 4.2 There is $\tau>0$ such that $\left\|\frac{u}{\|u\|}-e_{1}\right\| \geq \tau$ for all $u \in \mathcal{M}_{\mu}^{-}$with $\mu>0$.

Proof Suppose the contrary. There are $\tilde{\mu}_{n}$ and $u_{n} \in \mathcal{M}_{\tilde{\mu}_{n}}^{-}$such that $v_{n}:=\frac{u_{n}}{\left\|u_{n}\right\|} \rightarrow e_{1}$. Using the fact that

$$
0 \leq\left\|u_{n}\right\|^{2}-\tilde{\mu}_{n} \int|\xi|_{H}^{\alpha}\left|u_{n}\right|^{2} d \xi<\left(2^{\sharp}-1\right) \int K(\xi)\left|u_{n}\right|^{2^{\sharp}} d \xi,
$$

and the strong convergence of $v_{n}$ to $e_{1}$, we deduce that

$$
0 \leq\left\|v_{n}\right\|^{2}-\tilde{\mu}_{n} \int|\xi|_{H}^{\alpha}\left|v_{n}\right|^{2} d \xi<\left(2^{\sharp}-1\right)\left(\int K(\xi)\left|v_{n}\right|^{2^{\sharp}} d \xi\right)\left\|u_{n}\right\|^{2^{\sharp}-2} .
$$

Hence one obtains

$$
0 \leq\left(2^{\sharp}-1\right) \int K(\xi)\left|v_{n}\right|^{2^{\sharp}} d \xi \rightarrow\left(2^{\sharp}-1\right) \int K(\xi) e_{1}^{2^{\sharp}} d \xi<0,
$$

which is a contradiction.

Lemma 4.3 For $\tau$ given in Lemma 4.2, there is a $\mu_{* 1}>\mu_{1}$ such that $\|u\|^{2} \geq \mu_{* 1} \int|\xi|_{H}^{\alpha}|u|^{2} d \xi$ for any $u$ with $\|u\|=1$ and $\left\||u|-e_{1}\right\| \geq \tau$.

Proof Arguing by a contradiction, we assume that there are $\left\|u_{n}\right\|=1$ with $\left\|u_{n}-e_{1}\right\| \geq \tau$ and $\tilde{\mu}_{n} \rightarrow \mu_{1}$ with $\tilde{\mu}_{n}>\mu_{1}$ such that $\left\|u_{n}\right\|^{2}=\tilde{\mu}_{n} \int|\xi|_{H}^{\alpha}\left|u_{n}\right|^{2} d \xi$. Going if necessary to a subsequence, still denoted by $\left(u_{n}\right)_{n \in \mathbb{N}}$, we may assume that $u_{n} \rightarrow u_{0}$ in $S_{0}^{1,2}(\Omega)$ and therefore $u_{n} \rightarrow u_{0}$ in $L^{2}\left(\Omega,|\xi|_{H}^{\alpha} d \xi\right)$ (note that we have from Lemma 2.4 that $\int|\xi|_{H}^{\alpha}\left|u_{n}-u_{0}\right|^{2} d \xi \rightarrow 0$ as $n \rightarrow \infty)$. Combining this with $\tilde{\mu}_{n} \rightarrow \mu_{1}$ and $\|u\|^{2}-\mu_{1} \int|\xi|_{H}^{\alpha}|u|^{2} d \xi \geq 0$ for any $u \in$ $S_{0}^{1,2}(\Omega)$, we obtain

$$
\begin{aligned}
0 & \leq\left\|u_{0}\right\|^{2}-\mu_{1} \int|\xi|_{H}^{\alpha}\left|u_{0}\right|^{2} d \xi \\
& \leq \lim _{n \rightarrow \infty}\left(\left\|u_{n}\right\|^{2}-\tilde{\mu}_{n} \int|\xi|_{H}^{\alpha}\left|u_{n}\right|^{2} d \xi\right)=0 .
\end{aligned}
$$

If $u_{0}=0$, then we conclude from

$$
\left\|u_{n}\right\|^{2}=\tilde{\mu}_{n} \int|\xi|_{H}^{\alpha}\left|u_{n}\right|^{2} d \xi \rightarrow \mu_{1} \int|\xi|_{H}^{\alpha}\left|u_{0}\right|^{2} d \xi
$$


that $\left\|u_{n}\right\|^{2} \rightarrow 0$, which contradicts $\left\|u_{n}\right\|=1$. Assume $u_{0} \neq 0$, then (4.3) and the variational characterization of $\mu_{1}$ imply $u_{0}=t e_{1}$ for some $t \neq 0$. From

$$
\begin{aligned}
0 & \leq\left\|t e_{1}\right\|^{2}-\mu_{1} \int|\xi|_{H}^{\alpha}\left|t e_{1}\right|^{2} d \xi \leq \lim _{n \rightarrow \infty}\left(\left\|u_{n}\right\|^{2}-\tilde{\mu}_{n} \int|\xi|_{H}^{\alpha}\left|u_{n}\right|^{2} d \xi\right) \\
& =\lim _{n \rightarrow \infty}\left\|u_{n}\right\|^{2}-\mu_{1} \int|\xi|_{H}^{\alpha}\left|t e_{1}\right|^{2} d \xi=0
\end{aligned}
$$

we have $\lim _{n \rightarrow \infty}\left\|u_{n}\right\|^{2}=\left\|t e_{1}\right\|^{2}$. Hence

$$
\left\|u_{n}-t e_{1}\right\|^{2}=\left\|u_{n}\right\|^{2}-\left\|t e_{1}\right\|^{2}-2\left\langle u_{n}, t e_{1}\right\rangle \rightarrow 0 .
$$

It follows that $u_{n} \rightarrow t e_{1}$ and $t=1$. But this is impossible. The proof is complete.

Lemma 4.4 For any $\mu \in\left(\mu_{1}, \mu_{* 1}\right), \mathcal{M}_{\mu}^{-}$is closed in $S_{0}^{1,2}(\Omega)$ and open in $\mathcal{M}_{\mu}$.

Proof The openness in $\mathcal{M}_{\mu}$ is obvious. For the closedness, we argue by a contradiction. Suppose for $u_{n} \in \mathcal{M}_{\mu}^{-}, u_{n} \rightarrow u_{0}$ strongly in $S_{0}^{1,2}(\Omega)$ with $u_{0} \notin \mathcal{M}_{\mu}^{-}$. Then $u_{0} \in \mathcal{M}_{\mu}^{0}$, or equivalently $\int K(\xi)\left|u_{0}\right|^{2^{\sharp}} d \xi=0$. From $u_{n} \in \mathcal{M}_{\mu}^{-}$, we deduce that as $n \rightarrow \infty$,

$$
\begin{aligned}
0 & \leq\left\|u_{n}\right\|^{2}-\mu \int|\xi|_{H}^{\alpha}\left|u_{n}\right|^{2} d \xi<\left(2^{\sharp}-1\right) \int K(\xi)\left|u_{n}\right|^{2^{\sharp}} d \xi \\
& \rightarrow \int K(\xi)\left|u_{0}\right|^{2^{*}} d \xi=0 .
\end{aligned}
$$

Denote $v_{n}=u_{n} /\left\|u_{n}\right\|$ and divide (4.6) by $\left\|u_{n}\right\|^{2}$. Using the fact that $u_{n} \in \mathcal{M}_{\mu}^{-},\left\|v_{n}\right\|=1$ and Lemma 4.2, Lemma 4.3, we obtain

$$
0 \leq\left(\mu_{* 1}-\mu\right) \int|\xi|_{H}^{\alpha}\left|v_{n}\right|^{2} d \xi \leq\left\|v_{n}\right\|^{2}-\mu \int|\xi|_{H}^{\alpha}\left|v_{n}\right|^{2} d \xi \rightarrow 0
$$

It follows that $v_{n} \rightarrow 0$ strongly in $L^{2}\left(\Omega,|\xi|_{H}^{\alpha} d \xi\right)$. Therefore by (4.7), one gets $\left\|v_{n}\right\| \rightarrow 0$, which contradicts the fact that $\left\|v_{n}\right\|=1$.

Lemma 4.5 There is $\mu_{* 2}>\mu_{1}$ such that for any $\mu \in\left(\mu_{1}, \mu_{* 2}\right), \mathcal{M}_{\mu}^{+}$is bounded in $S_{0}^{1,2}(\Omega)$.

Proof Suppose the contrary, there are $\tilde{\mu}_{n}>\mu_{1}$ and $u_{n} \in \mathcal{M}_{\tilde{\mu}_{n}}^{+}$such that $\tilde{\mu}_{n} \rightarrow \mu_{1}$ and $\left\|u_{n}\right\| \rightarrow+\infty$ as $n \rightarrow \infty$. Note that $u_{n} \in \mathcal{M}_{\tilde{\mu}_{n}}^{+}$implies that

$$
\begin{aligned}
0 & >\left(\left\|u_{n}\right\|^{2}-\tilde{\mu}_{n} \int|\xi|_{H}^{\alpha}\left|u_{n}\right|^{2} d \xi\right)>\left(2^{\sharp}-1\right) \int K(\xi)\left|u_{n}\right|^{2^{\sharp}} d \xi \\
& =\left(2^{\sharp}-1\right)\left(\left\|u_{n}\right\|^{2}-\tilde{\mu}_{n} \int|\xi|_{H}^{\alpha}\left|u_{n}\right|^{2} d \xi\right) .
\end{aligned}
$$

Dividing (4.8) by $\left\|u_{n}\right\|^{2}$ and letting $v_{n}=u_{n} /\left\|u_{n}\right\|$, we obtain from $\tilde{\mu}_{n} \rightarrow \mu_{1}$

$$
\int K(\xi)\left|v_{n}\right|^{2^{\sharp}} d \xi \rightarrow 0 .
$$


On the other hand, from $\left\|v_{n}\right\|=1$, we may assume that there is a subsequence of $\left(v_{n}\right)_{n \in \mathbb{N}}$, still denoted by $\left(v_{n}\right)_{n \in \mathbb{N}}$ such that $v_{n} \rightarrow v_{0}$ weakly in $S_{0}^{1,2}(\Omega)$. Then using (4.8) and an argument similar to those in the proof of (4.3) that $v_{0}=t e_{1}$ for some $t \neq 0$. The same argument as in (4.4) and (4.5) lets us arrive at $v_{n} \rightarrow t e_{1}$ strongly in $S_{0}^{1,2}(\Omega)$. Thus as $n \rightarrow \infty$, we get

$$
\int K(\xi)\left|v_{n}\right|^{2^{\sharp}} d \xi \rightarrow \int K(\xi)\left|t e_{1}\right|^{2^{\sharp}} d \xi<0,
$$

which is a contradiction to (4.9). The proof is complete.

We are now in a position to prove the existence of one non-negative solution of (1.1) in the case of $\mu=\mu_{1}$.

Proof of (i) of Theorem 1.2 As pointed out in Remark 4.1, when $\mu=\mu_{1}, \mathcal{M}_{\mu_{1}}^{+}=\emptyset$. Hence we consider the minimization problem

$$
c_{2}=\inf _{u \in \mathcal{M}_{\mu_{1}}^{-}} L_{\mu_{1}}(u)
$$

Note that $\left(\left(\left\|\nu_{\lambda}\right\|^{2}-\mu \int|\xi|_{H}^{\alpha}\left|\nu_{\lambda}\right|^{2} d \xi\right) / \int K(\xi)\left|\nu_{\lambda}\right|^{2^{\sharp}}\right)^{(q-2) / 4} \nu_{\lambda} \in \mathcal{M}_{\mu_{1}}^{-}$, we can see from an argument similar to the proofs of Lemmas 3.2-3.4 that $c_{2}$ is achieved by some $w_{2}$. It then follows that $w_{2}$ is a solution of (1.1) with $\mu=\mu_{1}$. Moreover, $w_{2}$ can be chosen to be nonnegative. The proof is complete.

Next we turn to the case of $\mu>\mu_{1}$. Let $d_{1}=\inf _{u \in \mathcal{M}_{\mu}} L_{\mu}(u)$. From the previous lemma, $L_{\mu}$ is bounded from below on $\mathcal{M}_{\mu}^{+}$for $\mu \in\left(\mu_{1}, \mu_{* 2}\right)$. Since $t e_{1} \in \mathcal{M}_{\mu}^{+}$when $\mu>\mu_{1}$, the infimum of $L_{\mu}$ on $\mathcal{M}_{\mu}^{+}$must be negative. The characterization of $\mathcal{M}_{\mu}$ (see the beginning of Section 4) implies that $d_{1}=\inf _{u \in \mathcal{M}_{\mu}^{+}} L_{\mu}(u)$. Moreover, we have the following lemma.

Lemma 4.6 For $\mu_{1}<\mu<\min \left\{\mu_{* 1}, \mu_{* 2}\right\}, d_{1}$ is obtained by some $u_{*} \in \mathcal{M}_{\mu}^{+}$, which define a non-negative solution of (1.1).

Proof Similar to the previous proof, we know that there is $u_{*} \in \mathcal{M}_{\mu}$ such that $L_{\mu}\left(u_{*}\right)=d_{1}$. Moreover, $u_{*}$ solves (1.1) and can be chosen to be non-negative. Since $d_{1}<0$ and $L_{\mu}(u)=0$ for $u \in \mathcal{M}_{\mu}^{0}$ and $L_{\mu}(u)>0$ for $u \in \mathcal{M}_{\mu}^{-}$, we can conclude that $u_{*} \in \mathcal{M}_{\mu}^{+}$.

Let

$$
d_{2}=\inf _{u \in \mathcal{M}_{\bar{\mu}}^{\bar{\mu}}} L_{\mu}(u)
$$

Lemma 4.7 For $\mu_{1}<\mu<\min \left\{\mu_{* 1}, \mu_{* 2}\right\}$, there is a sequence $\left(u_{n}\right)_{n \in \mathbb{N}} \subset \mathcal{M}_{\mu}^{-}$such that $L_{\mu}\left(u_{n}\right) \rightarrow d_{2}, L_{\mu}^{\prime}\left(u_{n}\right) \rightarrow 0$, and if the $d_{2}<d_{1}+\frac{1}{q} \frac{S^{\frac{q}{2}}}{|K|_{\infty}^{(q-2) / 2}}$, then $\left(u_{n}\right)_{n \in \mathbb{N}}$ possesses a convergent subsequence in $S_{0}^{1,2}(\Omega)$.

Proof The idea of the proof is the same as [24], Lemma 4.7; see also [25]. We only outline the proof here. Similar to the proof in Lemma 3.2, there is a sequence $\left(u_{n}\right)_{n \in \mathbb{N}} \subset \mathcal{M}_{\mu}^{-}$such that

$$
L_{\mu}\left(u_{n}\right) \rightarrow d_{2}, \quad L_{\mu}^{\prime}\left(u_{n}\right) \rightarrow 0 \quad \text { in }\left(S_{0}^{1,2}(\Omega)\right)^{*}
$$


We first claim that $\left(u_{n}\right)_{n \in \mathbb{N}}$ is bounded in $S_{0}^{1,2}(\Omega)$. Indeed if $\left\|u_{n}\right\| \rightarrow \infty$, we denote $v_{n}=$ $u_{n} /\left\|u_{n}\right\|$, then $\left\|v_{n}\right\|=1$. From $u_{n} \in \mathcal{M}_{\mu}^{-}$, we have

$$
\begin{aligned}
0 & \leq \int K(\xi)\left|u_{n}\right|^{2^{\sharp}} d \xi \\
& =\left\|u_{n}\right\|^{2}-\mu \int|\xi|_{H}^{\alpha}\left|u_{n}\right|^{2} d \xi<\left(2^{\sharp}-1\right) \int K(\xi)\left|u_{n}\right|^{2^{\sharp}} d \xi .
\end{aligned}
$$

Dividing (4.11) by $\left\|u_{n}\right\|^{2}$, we get

$$
\begin{aligned}
0 & \leq\left(\mu_{* 1}-\mu\right) \int|\xi|_{H}^{\alpha}\left|v_{n}\right|^{2} d \xi \leq\left\|v_{n}\right\|^{2}-\mu \int|\xi|_{H}^{\alpha}\left|v_{n}\right|^{2} d \xi \\
& =\left\|u_{n}\right\|^{\|^{\sharp}-2} \int K(\xi)\left|v_{n}\right|^{2^{\sharp}} d \xi \rightarrow 0 .
\end{aligned}
$$

Therefore, $v_{n} \rightarrow 0$ strongly in $L^{2}\left(\Omega,|\xi|_{H}^{\alpha} d \xi\right)$ and hence $\left\|v_{n}\right\|^{2} \rightarrow 0$, which contradicts $\left\|v_{n}\right\|=1$. Thus $\left(u_{n}\right)_{n \in \mathbb{N}}$ is bounded in $S_{0}^{1,2}(\Omega)$.

Going if necessary to a subsequence, we may assume that $u_{n}$ converges to $u$ weakly in $S_{0}^{1,2}(\Omega)$ and almost everywhere in $\Omega$. Moreover, $\nabla_{H} u_{n} \rightarrow \nabla_{H} u$ a.e. in $\Omega$. Combining these with $L_{\mu}^{\prime}\left(u_{n}\right) \rightarrow 0$ we have $L_{\mu}^{\prime}(u)=0$. In particular, we have $u \in \mathcal{M}_{\mu}$. Hence

$$
L_{\mu}(u)=\frac{1}{q}\left(\|u\|^{2}-\mu \int|\xi|_{H}^{\alpha}|u|^{2} d \xi\right) \geq d_{1}
$$

If $u_{n} \rightarrow u$ strongly in $S_{0}^{1,2}(\Omega)$, then we complete the proof. If $u_{n}$ does not converge strongly to $u$ in $S_{0}^{1,2}(\Omega)$, then we denote $\tilde{u}_{n}=u_{n}-u$. From $L_{\mu}^{\prime}\left(u_{n}\right)=0$, we can deduce that, for $n$ large enough,

$$
\int\left|\nabla_{H} \tilde{u}_{n}\right|^{2} d \xi-\int K(\xi)\left|\tilde{u}_{n}\right|^{2^{\sharp}} d \xi=o(1) .
$$

Suppose that $\int\left|\tilde{u}_{n}\right|^{2^{\sharp}} d \xi \not \nrightarrow 0$ as $n \rightarrow \infty$, we may deduce from the Sobolev inequality (1.2) that

$$
L_{\mu}\left(\tilde{u}_{n}\right) \geq \frac{1}{q} \frac{S^{\frac{q}{2}}}{|K|_{\infty}^{(q-2) / 2}} .
$$

Therefore we obtain from the Brezis-Lieb lemma again for $n$ large enough

$$
\begin{aligned}
d_{2}+o(1) & =L_{\mu}\left(u_{n}\right) \geq \frac{1}{q}\left(\|u\|^{2}-\mu \int|\xi|_{H}^{\alpha}|u|^{2} d \xi\right)+L_{\mu}\left(\tilde{u}_{n}\right)+o(1) \\
& \geq d_{1}+\frac{1}{q} \frac{S^{q / 2}}{|K|_{\infty}^{(q-2) / 2}}
\end{aligned}
$$

which is a contradiction. Thus we can conclude that $u_{n} \rightarrow u$ strongly in $S_{0}^{1,2}(\Omega)$.

Lemma 4.8 There is $\mu_{*}>\mu_{1}$ such that for any $\mu \in\left(\mu_{1}, \mu_{*}\right)$, the $d_{2}<d_{1}+\frac{1}{q} \frac{S^{\frac{q}{2}}}{|K|_{\infty}^{(q-2) / 2}}$. 
In order to prove Lemma 4.8, we need some further lemmas, which play a key role in the proof of Lemma 4.8. It is Lemma 4.9 and Lemma 4.10 that we need to address the regularity for the solution of (1.1).

Lemma 4.9 Let $w$ be a non-negative solution of (1.1). If $0<\alpha<\frac{q}{2}-3$, then for $\lambda$ large enough,

$$
\int w_{1}^{2^{\sharp}-1} v_{\lambda} d \xi=o\left(\lambda^{-\frac{q-2}{2}}\right) \quad \text { and } \quad \int w_{1}\left(v_{\lambda}\right)^{2^{\sharp}-1} d \xi=o\left(\left(\lambda^{-1}\right)^{2+\alpha}\right) \text {. }
$$

Proof Since $w_{1} \in L^{r}(\Omega)$ for any $r \in(1, \infty)$, we obtain from the Hölder inequality

$$
\int w_{1}^{2^{\sharp}-1} v_{\lambda} d \xi \leq\left(\int v_{\lambda}^{\gamma} d \xi\right)^{\frac{1}{\gamma}}\left(\int w_{1}^{\frac{\left(2^{\sharp}-1\right) \gamma}{\gamma-1}} d \xi\right)^{\frac{\gamma-1}{\gamma}}
$$

where $\gamma>1$ and $q>(q-2) \gamma$. Note that

$$
\begin{aligned}
\int v_{\lambda}^{\gamma} d \xi & =\int_{|\xi|_{H<2 R}}\left(w_{\lambda}(\xi)\right)^{\gamma} d \xi=\lambda^{\frac{(q-2) \gamma}{2}} \int_{|\xi|_{H}<2 R}\left(w\left(\delta_{\lambda}(\xi)\right)\right)^{\gamma} d \xi \\
& =\lambda^{\frac{(q-2) \gamma}{2}-q} \int_{|\eta|_{H}<2 \lambda R}(w(\eta))^{\gamma} d \eta \\
& =\lambda^{\frac{(q-2) \gamma}{2}-q}\left(C+\int_{1}^{2 \lambda R} \rho^{-1+q-(q-2) \gamma} d \rho\right) .
\end{aligned}
$$

From the choice of $\gamma$, we have

$$
\int v_{\lambda}^{\gamma} d \xi=C \cdot \lambda^{\frac{q-2}{2} \gamma-q}+C \cdot \lambda^{-\frac{q-2}{2} \gamma}
$$

Therefore as $\lambda$ is sufficiently large, one deduces that

$$
\int w_{1}^{2^{\sharp}-1} v_{\lambda} d \xi=o\left(\lambda^{-\frac{q-2}{2}}\right) .
$$

Similarly, we can use the regularity of $w_{1}$ to prove that as $\lambda$ is large enough, there is $\beta$ with $1<\beta<\frac{2 q}{q+2+2(2+\alpha)}$ such that

$$
\int w_{1}\left(v_{\lambda}\right)^{2^{\sharp}-1} d \xi=C \cdot\left(\lambda^{-1}\right)^{\frac{q}{\beta}-\frac{q+2}{2}},
$$

where we have used the assumption $0<\alpha<\frac{q}{2}-3$. Therefore as $\lambda$ is sufficiently large, one has

$$
\int w_{1}\left(v_{\lambda}\right)^{2^{\sharp}-1} d \xi=o\left(\left(\lambda^{-1}\right)^{2+\alpha}\right) .
$$

The proof is complete.

Lemma 4.10 Let $w$ be a non-negative solution of (1.1). Then there are $s_{0}>0$ and $\tilde{\mu}>\mu_{1}$ such that $w+s_{0} \nu_{\lambda} \in \mathcal{M}_{\mu}^{-}$for all $0<\mu<\tilde{\mu}$. 
Proof For any $s>0$, since $G(w)=0$ and $w$ satisfies (1.1), we have

$$
\begin{aligned}
G\left(w+s v_{\lambda}\right)= & G\left(s v_{\lambda}\right)+2 s \int K(\xi) w^{2^{\sharp}-1} v_{\lambda} d \xi \\
& +\int K(\xi)\left(w^{2^{\sharp}}+\left(s v_{\lambda}\right)^{2^{\sharp}}-\left|w+s v_{\lambda}\right|^{2^{\sharp}}\right) d \xi .
\end{aligned}
$$

Using the elementary inequality

$$
|a+b|^{p} \geq|a|^{p}+|b|^{p}-M\left(|a|^{p-1}|b|+|a||b|^{p-1}\right), \quad \forall p>1, a, b \in \mathbb{R}
$$

and the fact that $K(\xi)$ is bounded in $\Omega$, we obtain

$$
\begin{aligned}
& \left|\int K(\xi)\left(w^{2^{\sharp}}+\left(s v_{\lambda}\right)^{2^{\sharp}}-\left|w+s v_{\lambda}\right|^{2^{\sharp}}\right) d \xi\right| \\
& \quad \leq C s \int w^{2^{\sharp}-1} v_{\lambda} d \xi+C s^{2^{\sharp}-1} \int w v_{\lambda}^{2^{\sharp}-1} d \xi .
\end{aligned}
$$

Therefore for any finite $s$, we obtain from (2.2) and Lemma 4.9

$$
\begin{aligned}
G\left(w+s v_{\lambda}\right) & =G\left(s v_{\lambda}\right)+o\left(\left(\lambda^{-1}\right)^{(2+\alpha)}\right) \\
& =s^{2} \int\left|\nabla_{H} v_{\lambda}\right|^{2} d \xi-s^{2^{\sharp}} \int K(\xi) v_{\lambda}^{2^{\sharp}} d \xi-o\left(\lambda^{-(2+\alpha)}\right)
\end{aligned}
$$

for $\lambda$ large enough. Thus there is $s_{0}>0$ such that $G\left(w+s_{0} v_{\lambda}\right)=0$, which implies that $w+$ $s_{0} v_{\lambda} \in \mathcal{M}_{\mu}$.

Next, to see $w+s_{0} v_{\lambda} \in \mathcal{M}_{\mu}^{-}$, it suffices to prove that

$$
\int K(\xi)\left|w+s_{0} v_{\lambda}\right|^{2^{\sharp}} d \xi>0 \text { for } \lambda \text { large enough. }
$$

Indeed, using inequality (4.13) and Lemma 4.9, we obtain

$$
\begin{aligned}
\int & K(\xi)\left|w+s_{0} v_{\lambda}\right|^{2^{\sharp}} d \xi \\
& =\int K(\xi) w^{2^{\sharp}} d \xi+s_{0}^{2^{\sharp}} \int K(\xi) v_{\lambda}^{2^{\sharp}} d \xi+o\left(\left(\lambda^{-1}\right)^{2+\alpha}\right) \\
& =\int\left(\left|\nabla_{H} w\right|^{2}-\mu|\xi|_{H}^{\alpha} w^{2}\right) d \xi+s_{0}^{2^{\sharp}} \int K(\xi) v_{\lambda}^{2^{\sharp}} d \xi+o\left(\left(\lambda^{-1}\right)^{2+\alpha}\right) \\
\geq & \left(1-\frac{\mu}{\mu_{1}}\right) \int\left|\nabla_{H} w\right|^{2} d \xi+s_{0}^{2^{\sharp}} \int K(\xi) v_{\lambda}^{2^{\sharp}} d \xi+o\left(\left(\lambda^{-1}\right)^{2+\alpha}\right)
\end{aligned}
$$

for $\lambda$ large enough. It follows from $G\left(w+s_{0} \nu_{\lambda}\right)=0$ that there is $\tilde{\mu}>\mu_{1}$ such that

$$
\int K(\xi)\left|w+s_{0} v_{\lambda}\right|^{2^{\sharp}} d \xi>0, \quad 0<\mu<\tilde{\mu} .
$$

The proof is complete. 
Proof of Lemma 4.8 Using the fact that $L_{\mu}\left(u_{*}\right)=d_{1}, u_{*}$ satisfies (1.1) and (4.13), Lemma 4.9, we obtain from a direct computation for $\lambda$ large enough

$$
L_{\mu}\left(u_{*}+s v_{\lambda}\right) \leq L_{\mu}\left(u_{*}\right)+L_{\mu}\left(s v_{\lambda}\right)+o\left(\left(\lambda^{-1}\right)^{2+\alpha}\right)
$$

In view of Lemma 4.10, it suffices to prove that

$$
\sup _{s>0} L_{\mu}\left(u_{*}+s v_{\lambda}\right)<d_{1}+\frac{1}{q} \frac{S^{\frac{q}{2}}}{|K|_{\infty}^{\frac{q-2}{2}}} .
$$

Note that

$$
\begin{aligned}
& \sup _{s>0} L_{\mu}\left(s v_{\lambda}\right) \\
& \quad=\frac{1}{q}\left(\left\|v_{\lambda}\right\|^{2}-\mu \int|\xi|_{H}^{\alpha}\left|v_{\lambda}\right|^{2} d \xi\right)^{\frac{q}{2}}\left(\int K(\xi)\left|v_{\lambda}\right|^{2^{\sharp}} d \xi\right)^{\frac{2-q}{2}} \\
& \quad=\frac{1}{q}\left(S^{\frac{q}{2}}+O\left(\left(\lambda^{-1}\right)^{q-2}\right)-O\left(\left(\lambda^{-1}\right)^{\alpha+2}\right)\right)^{\frac{q}{2}}\left(|K|_{\infty} S^{\frac{q}{2}}+O\left(\left(\lambda^{-1}\right)^{\beta}\right)+O\left(\left(\lambda^{-1}\right)^{q}\right)\right)^{\frac{2-q}{2}} \\
& \quad=\frac{1}{q} \frac{S^{\frac{q}{2}}}{|K|_{\infty}^{\frac{q-2}{2}}}-O\left(\lambda^{-(2+\alpha)}\right)+o\left(\lambda^{-\frac{q-2}{2}}\right)
\end{aligned}
$$

for $\lambda$ large enough. Denote $\mu_{*}=\min \left\{\mu_{* 1}, \mu_{* 2}, \tilde{\mu}\right\}$. Then one has

$$
\begin{aligned}
\sup _{t>0} L_{\mu}\left(u_{*}+t v_{\lambda}\right)= & d_{1}+\frac{1}{q} \frac{S^{\frac{q}{2}}}{|K|_{\infty}^{\frac{q-2}{2}}}-O\left(\lambda^{-(2+\alpha)}\right) \\
& +o\left(\lambda^{-(2+\alpha)}\right)+O\left(\lambda^{-\frac{q-2}{2}}\right) \\
& <d_{1}+\frac{1}{q} \frac{S^{\frac{q}{2}}}{|K|_{\infty}^{\frac{q-2}{2}}} .
\end{aligned}
$$

Proof of (ii) of Theorem 1.2 The proof is a combination of Lemma 4.6, Lemma 4.7, Lemma 4.8, and the fact that if $\left(u_{n}\right)_{n \in \mathbb{N}}$ is a minimizing sequence of $d_{2}$, then so is $\left(\left|u_{n}\right|\right)_{n \in \mathbb{N}}$. The proof is complete.

Competing interests

The authors declare that they have no competing interests.

\section{Authors' contributions}

The authors declare that the study was realized in collaboration with the same responsibility. All authors read and approved the final manuscript.

\section{Author details}

${ }^{1}$ College of Mathematics and Physics, Fujian Jiangxia University, Fuzhou, 350108, P.R. China. ${ }^{2}$ School of Mathematics and Computer Science, Fujian Normal University, Fuzhou, 350108, P.R. China. ${ }^{3}$ Center for Research \& Development in Mathematics and Applications, University of Aveiro, Aveiro, 3810-193, Portugal.

\section{Acknowledgements}

The authors thank the referee for kindly pointing out $[1,8,12-15,22]$. L. Huang is partially supported by NSF of China (No. 11501107). J. Chen was supported by NSF of China (No. 11371091) and the innovation group of 'Nonlinear analysis and its applications' (No. IRTL1206). E. Rocha was supported by Portuguese funds through the CIDMA of University of Aveiro and the Portuguese Foundation for Science and Technology. 


\section{References}

1. Bonfiglioli, A, Lanconelli, E, Uguzzoni, F: Stratified Lie Groups and Potential Theory for Their Sub-Laplacians. Springer Monograph in Mathematics. Springer, Berlin (2007)

2. Folland, GB, Stein, EM: Estimates for the d complex and analysis on the Heisenberg group. Commun. Pure Appl. Math. 27, 429-522 (1974)

3. Folland, GB, Stein, EM: Hardy Spaces on Homogeneous Groups. Math. Notes, vol. 28. Princeton University Press, Princeton (1982)

4. Citti, G: Semilinear Dirichlet problem involving critical exponent for the Kohn Laplacian. Ann. Mat. Pura Appl. CLXIX 375-392 (1995)

5. Birindelli, I, Capuzzo Dolcetta, I, Cutri, A: Indefinite semi-linear equations on the Heisenberg group: a priori bounds and existence. Commun. Partial Differ. Equ. 23, 1123-1157 (1998)

6. Birindelli, I, Cutri, A: A semi-linear problem for the Heisenberg Laplacian. Rend. Semin. Mat. Univ. Padova 94, 137-153 (1995)

7. Uguzzoni, F: A non-existence theorem for a semilinear Dirichlet problem involving critical exponent on halfspaces of the Heisenberg group. Nonlinear Differ. Equ. Appl. 6, 191-206 (1999)

8. Uguzoni, F: A note on Yamabe-type equations on the Heisenberg group. Hiroshima Math. J. 30, 179-189 (2000)

9. Brandolini, L, Rigoli, M, Setti, AG: Positive solutions of Yamabe-type equations on the Heisenberg group. Duke Math. J. 91, 241-296 (1998)

10. Jerison, DS, Lee, JM: Intrinsic CR coordinates and the CR Yamabe problem. J. Differ. Geom. 29, 303-343 (1981)

11. Garofalo, N, Lanconelli, E: Existence and non existence results for semilinear equations on the Heisenberg group. Indiana Univ. Math. J. 41, 71-97 (1992)

12. Felli, V, Uguzzoni, F: Some existence results for the Webster scalar curvature problem in presence of symmetry. Ann. Mat. Pura Appl. 183, 469-493 (2004)

13. Malchiodi, A, Uguzzoni, F: A perturbation result for the Webster scalar curvature problem on the CR sphere. J. Math. Pures Appl. 81, 983-997 (2002)

14. Lanconelli, E, Uguzzoni, F: Asymptotic behavior and non-existence theorems for semilinear Dirichlet problems involving critical exponent on unbounded domains of the Heisenberg group. Boll. Unione Mat. Ital. 8(1-B), 139-168 (1998)

15. Lanconelli, E, Uguzzoni, F: Non-existence results for semilinear Kohn-Laplace equations in unbounded domains. Commun. Partial Differ. Equ. 25, 1703-1739 (2000)

16. Citti, G, Uguzzoni, F: Critical semilinear equations on the Heisenberg group: the effect of topology of the domain. Nonlinear Anal. 46, 399-417 (2001)

17. Folland, GB. Subelliptic estimates and function spaces on nilpotent Lie groups. Ark. Mat. 13, 161-207 (1975)

18. Garofalo, N, Nhieu, D: Isoperimetric and Sobolev inequalities for Carnot-Caratheodory spaces and the existence of minimal surfaces. Commun. Pure Appl. Math. XLIX, 1081-1144 (1996)

19. Garofalo, N, Vassilev, D: Regularity near the characteristic set in the non-linear Dirichlet problem and conformal geometry of sub-Laplacians on Carnot groups. Math. Ann. 318, 453-516 (2000)

20. Lanconelli, E: Critical semilinear equations on the Heisenberg group. Ann. Univ. Ferrara Sez. VII Sci. Mat. Suppl. XLV, 187-195 (1999)

21. Han, Y, Niu, PC: Hardy-Sobolev type inequalities on the H-type group. Manuscr. Math. 118, 235-252 (2005)

22. Garagnani, E, Uguzzoni, F: A multiplicity result for a degenerate-elliptic equation with critical growth on noncontractible domains. Topol. Methods Nonlinear Anal. 22, 53-68 (2003)

23. Jerison, DS, Lee, JM: Extremals of the Sobolev inequality on the Heisenberg group and the CR Yamabe problem. J. Am. Math. Soc. 1, 1-13 (1988)

24. Chen, J: Multiplicity result for a singular elliptic equation with indefinite nonlinearity. J. Math. Anal. Appl. 337, 493-504 (2008)

25. Drabek, P, Huang, YX: Multiplicity of positive solutions for some quasilinear elliptic equation in $\mathbb{R}^{N}$ with critical Sobolev exponent. J. Differ. Equ. 140, 106-132 (1997)

26. Brezis, $H$, Nirenberg, L: Positive solutions of nonlinear elliptic equations involving critical exponents. Commun. Pure Appl. Math. 34, 437-477 (1983)

27. Brezis, H, Kato, T: Remarks on the Schrödinger operator with singular complex potentials. J. Math. Pures Appl. 58 , 137-151 (1979)

28. Struwe, M: Variational Methods, Applications to Nonlinear Partial Differential Equations and Hamiltonian Systems, 2nd edn. Springer, Berlin (1996)

29. Mokrani, H: Semi-linear sub-elliptic equations on the Heisenberg group with a singular potential. Commun. Pure Appl. Anal. 8, 1619-1636 (2009)

30. Chen, J, Li, SJ, Li, YQ: Multiple solutions for a semilinear equation involving singular potential and critical exponent. Z. Angew. Math. Phys. 56, 453-474 (2005) 